\section{P305 CHALLENGES OF DEVELOPING CLINICAL PRACTICE GUIDELINES (CPG) FOR PEDIATRIC CANCER IN COLOMBIA}

A Linares, M Torres, R Pardo, Leukemia and Lymphoma, Guidelines Development Group. Clinical Research Institute, Universidad Nacional de Colombia, Bogota, Colombia

\section{0:1136/bmjqs-2013-002293.247}

Background WHO estimates for the year 2013 that approximately 12 million deaths will be attributed to cancer. In developing countries, 180.000 childhood cancer cases occur every year. Context the need to standardise the management of childhood haematological cancers and reach the same survival rates in developed and developing countries require the elaboration of clinical practice guidelines (CPG) in Colombia. The overall survival for acute lymphoid leukaemia is around 50\% while in other countries is near $80 \%$. description of best practice: the ministry of health assigned the development of a CPG of childhood acute leukemias and lymphomas. The guideline development group (GDG) had to face some challenges due to the amount/quality of evidence and the patients' point of view. lessons for guideline developers, adaptors, implementers, and/or users: during the formulation of the recommendations, not only effectiveness is a critical outcome but treatment toxicity and adverse effects such as secondary neoplasies need to be evaluated. In order to identify relevant information of safety, the GDG had to include observational studies which brought up some challenges. The GDG developed a qualitative exercise for the identification of patients' point of view that were weighted in equal manner with the risk and benefits of the interventions. Once the guideline was finished, the recommendations were driven towards patient safety and the implementation implied to make changes in public policies, improve the access to continuous therapy, proper diagnosis, modifications in resources assignment and work around the available treatment so the overall survival can reach international standards.

\section{P307 GUIDELINE DEVELOPMENT TOOL (GDT) - WEB-BASED SOLUTION FOR GUIDELINE DEVELOPERS AND AUTHORS OF SYSTEMATIC REVIEWS}

${ }^{2}$ J Brozek, ${ }^{1}$, E Akl, ${ }^{3} Y$ Falck-Ytter, ${ }^{4} \mathrm{P}$ Kunstman, ${ }^{5} \mathrm{~J}$ Meerpohl, ${ }^{1} \mathrm{R}$ Mustafa, ${ }^{4} \mathrm{~A}$ Nowak, ${ }^{6} \mathrm{~A}$ Oxman, ${ }^{1} \mathrm{~N}$ Santesso, ${ }^{1} \mathrm{~W}$ Wiercioch, ${ }^{1} \mathrm{H}$ Schünemann. ${ }^{1}$ McMaster University, Hamilton, Canada; ${ }^{2}$ American University of Beirut, Beirut, Lebanon; ${ }^{3}$ Case Western Reserve University, Cleveland, USA; ${ }^{4}$ Synaway, Krakow, Poland; ${ }^{5}$ Universitätsklinikum Freiburg, Freiburg, Germany Norwegian Knowledge Centre for the Health Services, Oslo, Norway

\section{0:1136/bmjqs-2013-002293.248}

Background Guideline developers and other health care decision makers benefit from following a structured process of specifying the health care questions they intend to answer and the outcomes of interest, assessing the confidence in the available evidence, gathering information about the values and preferences of the target population, and presentation of their results and decisions to the target users. Many guideline developers use the GRADE Profiler (GRADEpro) software used to conduct this work.

Context GRADE's approach is currently being further defined in the DECIDE (Developing and Evaluating Communication Strategies to Support Informed Decisions and Practice Based on Evidence) project.

Description of Best Practice The Guideline Development Tool (GDT) is the extension of the GRADE Profiler (GRADEpro) software. The GDT provides an integrated platform-independent web-based solution for health care decision makers offering support for the whole process of making decisions and developing recommendations including question formulation, generation and prioritisation of outcomes, support for teamwork, management of potential conflicts of interest, presentation of results (including the functionality of GRADEpro) and decision support. We tested the software with individual users and in workshops as well as in guideline development processes.

Lessons for Guideline Developers, Adaptors, Implementers, and/ or Users Following a structured and systematic process, transparency and clarity of presentation facilitates the use of results of systematic reviews and facilitates development, updating and adaptation of evidence-based recommendations and decisions. Storing all information in a uniform, structured, transparent and annotated way also greatly facilitates updating and adaptation of systematic reviews and guidelines.

\section{P308 DATA DRIVEN BEST PRACTICE IN GENERAL PRACTICE PRESCRIBING}

N Huang. MedicineWise

10:1136/bmjqs-2013-002293.249

Background A national quality improvement programme to understand how medicines are used was launched in 2011. The programme collects clinical data from general practice electronic health records to identify prescribing/management gaps.

Context The delivery of primary care is complex. The majority of medicines are prescribed by GPs. There is huge potential to use routinely collected clinical information to reduce evidencetreatment gaps and improve population health outcomes.

Description of Best Practice A scan of the literature and keynote international and national agencies is undertaken to identify the range of interventions and tools that support change in general practice prescribing. Activities reported as most effective, value for money and applicable to general practice, with a focus on those using prescribing or clinical data as key inputs are collated (including which activities were used singly or in combination). These activities are entered into an overarching intervention framework for data driven activities in general practice. A group of local clinical experts are invited to identify the barriers and enablers to apply this framework to the local primary care sector. Three topic areas are selected to model these approaches and verify the elements of the framework. An implementation plan including evaluation of effectiveness will be presented.

Lessons for Guideline Developers, Adaptors, Implementers, and/ or Users The design of the intervention framework illustrates ways to design an 'implementation strategy' that draws on three dimensions:literature on effectiveness of implementation interventions; applied experience of clinical leaders; local context of primary care.

\section{P309 SERIOUS GAMES AS A NEW MEDIUM FOR PATIENTS GUIDELINES DISSEMINATION}

${ }^{1} \mathrm{G}$ Georg, ${ }^{2} \mathrm{M}$ Cavazza, ${ }^{2} \mathrm{~F}$ Charles, ${ }^{2} \mathrm{C}$ Smith. ${ }^{1}$ Haute Autorité de Santé, Saint-Denis La Plaine, France; ${ }^{2}$ Teesside University, Middlesbrough, UK

\section{0:1136/bmjqs-2013-002293.250}

Background Patient education documents are faced with a number of issues that limit their dissemination, from intrinsic 